\title{
AVALIAÇÃO EXERGÉTICA DOS EFLUENTES DO PROCESSO INDUSTRIAL
} DO ÁLCOOL

Dissertação apresentada à Escola de Engenharia de São Carlos da Universidade de São Paulo, como parte dos requisitos para obtenção do Título de Mestre em Hidráulica e Saneamento

Orientador : Prof. Dr. Woodrow Nelson Lopes Roma 


\section{AGRADECIMENTOS}

Neste longo caminho muitas pessoas foram importantes, mas algumas se destacam pela motivação constante para finalização deste trabalho.

Ao Prof. Dr. Woodrow Nelson Lopes Roma, meu orientador, paciente, generoso, sábio, pela condução da orientação culminando com esta dissertação em uma área inovadora e muito promissora ainda.

Ao Dr Aldo Roberto Ometto, pelos aconselhamentos no decorrer dos seus estudos de doutoramento.

Ao engenheiro químico Guilherme Leiria Filho por ter me auxiliado na compreensão do processo industrial do álcool etílico carburante e em correções do processo.

Aos meus pais, José Hermínio Albuquerque e Maristela da Silva Albuquerque pela constância, amor e apoio estando sempre ao meu lado.

Minhas filhas, Agnes Afrodite, Isis Penélope e Ariadne Palas que mesmo num sutil acompanhamento me deram as forças para prosseguir neste trabalho.

A Rose Rubini, companheira, pessoa especial, que com seu incentivo, união de forças e dedicação esteve ao meu lado nesta empreitada, os mais efusivos agradecimentos. 
"Em todas as operações da natureza, nada se cria: a qualidade e quantidade dos elementos permanecem precisamente os mesmos, antes $e$ depois de um experimento. O que muda são suas combinações. Deste primeiro principio depende toda a arte de executar experiências químicas” Antoine Laurent Lavoisier (1743-1794) 


\section{RESUMO}

ALBUQUERQUE, A.G.(2005)- Avaliação exergética dos efluentes no processo industrial do álcool. Dissertação (Mestrado) apresentada na Escola de Engenharia de São Carlos, Universidade de São Paulo, São Carlos, 2005.

A indústria sucroalcooleira tem na produção do álcool etílico carburante a captação de água em grande volume para geração de vapor, resfriamento e como parte do processo, sendo também geradora de grandes quantidades de efluentes. A indústria utilizando a energia proveniente da queima do bagaço nas caldeiras para transformação da água em vapor, gera sua própria eletricidade, e todo trabalho mecânico desenvolvido no processo. $\mathrm{O}$ grau de aproveitamento do vapor residual em trocadores de calor demonstra a eficiência da empresa. O balanço das massas que estão sendo transformadas neste processo, permite avaliar através da temperatura dos efluentes lançados, as perdas de exergia que podem ser minoradas. A ferramenta análise exergética (medida do grau de desequilíbrio energético entre uma substância e seu ambiente) empregada neste trabalho, mostra através de estudo de caso em duas usinas, como identificar, na avaliação dos efluentes lançados as perdas exergéticas em volumes de controle traçados no ciclo de produção do álcool, onde estão acontecendo as perdas e as possibilidades de melhorias que venham a corrigi-las.

Palavras chaves: exergia, efluentes líquidos, sucroalcooleira, cana-de-açúcar, álcool etílico. 


\section{ABSTRACT}

ALBUQUERQUE, A.G.(2005) - Exergetic assessment of effluents in the industrial alcohol process. Master's Thesis presented at the São Carlos Engineering School, University of São Paulo, São Carlos, 2005.

In the production of ethyl alcohol as a fuel, the sugar and alcohol industry captures large volumes of water for steam generation, for cooling and as part of the process, generating large amounts of effluents. Using the energy produced by the burning of bagasse in the furnace for the transformation of water into steam, the industry generates its own electricity and the entire mechanical work developed in the process. The degree of utilization of residual steam in heat exchangers demonstrates the efficiency of the plant. The balance of the energies being transformed in this process shows, through the effluents launched, the losses that may be reduced. By means of case studies in two mills, the exergetic analysis tool ( measurement of the degree of unbalance between the substance and its environment) employed in this study shows how to identify, in control volumes traced in the alcohol production cycle, where the losses are occurring and the possibilities of improvement that may eventually correct them.

Key words - exergy, liquid effluents, sugar and alcohol, sugar cane, ethylic alcohol. 


\section{LISTA DE ILUSTRAÇÕES}

FIGURA 01- FLUXO DE PRODUÇÃO DO ÁlCOOL A PARTIR DA CANA-DEAÇÚCAR. 12

FIGURA 02- ESQUEMAS DE EMBEBIÇÃO UTILIZADOS NAS INDÚSTRIAS CANAVIEIRAS. 16

FIGURA 03- FLUXOGRAMA DESTILARIA. .27

FIGURA 04- MODELO DEMONSTRATIVO DA TROCA DE EXERGIA TÉRMICA ENTRE DOIS ESTADOS. 31

FIGURA 05- FLUXOGRAMA DE PRODUÇÃO DO ÁLCOOL COM INDICAÇÃO DOS VOLUMES DE CONTROLE A SEREM ANALISADOS. .36

FIGURA 06 -PROCESSO DE PRODUÇÃO DE ÁLCOOL NA UNIDADE I............ 38

FIGURA 07- FLUXOGRAMA DAS ÁGUAS DA UNIDADE I ...................................47

FIGURA 08- FLUXOGRAMA DAS ÁGUA UNIDADE II .52 


\section{LISTA DE TABELAS}

TABELA 01- Avaliação exergética aplicada ao ponto de descarte 1 da unidade I........ 54

TABELA 02- Avaliação exergética aplicada ao ponto de descarte 2 da unidade I........ 55

TABELA 03- Avaliação exergética aplicada ao ponto 1 da unidade II........................ 55

TABELA 04 - Avaliação exergética aplicada ao ponto 2 da unidade II..................... 55

\section{LISTA DE QUADROS}

QUADRO 1 - Produção nacional de cana-de-açúcar. 09 


\section{LISTA DE ABREVIATURAS E SIGLAS}

ABNT Associação Brasileira de Normas Técnicas

ART - Açúcar redutor total

ARC- açúcares redutores da cana

CNTP - Condições Normais de Temperatura e Pressão

CONSECANA - Conselho dos produtores de cana, açúcar e álcool do Estado de São

Paulo

COPERSUCAR - Cooperativa dos produtores de açúcar e álcool do Estado de São Paulo

DBO - Demanda Bioquímica de Oxigênio

FIPE - Fundação Instituto de Pesquisas Econômicas

IEA - Instituto de Economia Agricola de São Paulo

IPT - Instituto de Pesquisas Tecnológicas

PBU - Peso do bagaço úmido

PBS - peso do bagaço seco

OD - Oxigênio Dissolvido 


\section{LISTA DE SÍMBOLOS}

${ }^{\circ}$ Bé- grau Baumé

Brix - simbolo ${ }^{\circ}$ Brix, é a medida dos sólidos dissolvidos num líquido

$\mathrm{pH}$ - potencial hidrogeniônico

pol - percentual de sacarose aparente no caldo

$\mathrm{Kgv}$ - quilograma de vapor

$\mathrm{kJ}$ - quiloJoule

tc - tonelada de cana

tv - tonelada de vapor

$\mathrm{T}_{0}$ - temperatura ambiente de referência

$\mathrm{P}_{0}$ - pressão ambiente de referência

$\mathrm{H}$ - entalpia

$\mathrm{S}-$ entropia

$\mathrm{Q}_{0}$ - quantidade de calor transferida

$\mathrm{B}_{\mathrm{ch}}$ - exergia química

$\mathrm{B}_{\mathrm{ph}}$ - exergia física

$\mathrm{H}_{\mathrm{u}}$ - entalpia útil

$\mathrm{S}_{\mathrm{u}}$ - entropia útil

$\mathrm{H}_{\mathrm{ur}}$ - entalpia útil do processo reversível

$\mathrm{S}_{\mathrm{ur}}$ - entropia útil do processo reversível

$\mathrm{Q}_{0 \mathrm{r}}$ - quantidade de calor perdida no processo reversível 
$\sum$ - somatória

$\Delta S$ - variação da entropia

$\mathrm{c}_{\mathrm{w}}$ - capacidade de calor específico da água

$\mathrm{m}-$ massa

$\mathrm{T}_{\mathrm{s}}$ - temperatura de saída

$T_{e}$ - temperatura de entrada

$\mathrm{b}_{\mathrm{fT}}-$ exergia física à capacidade térmica constante

$\mathrm{c}_{\mathrm{p}}$ - capacidade térmica específica

$\mathrm{B}_{\mathrm{p}}-$ exergia a pressão constante

$\mathrm{V}_{1}$ - vapor vegetal 


\section{SUMÁRIO}

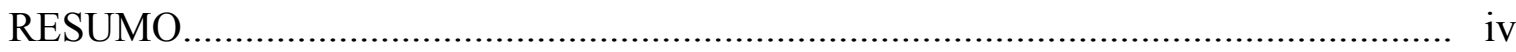

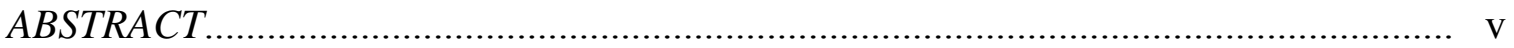

LISTA DE ILUSTRAÇÕES.............................................................................. vi

LISTA DE TABELAS................................................................................... vii

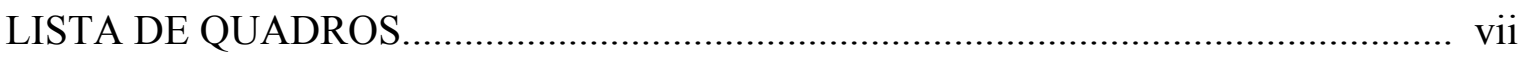

LISTA DE ABREVIATURA E SIGLAS............................................................ viii

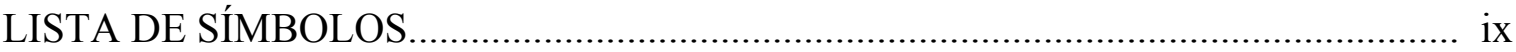

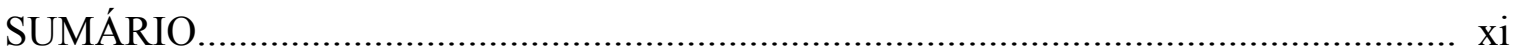

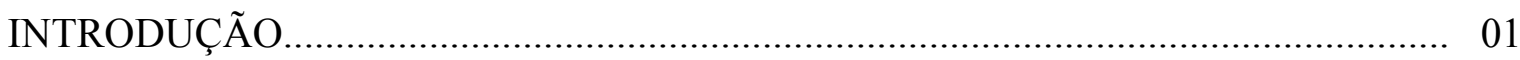

2. OBJETIVO

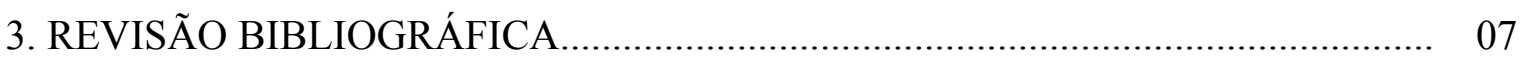

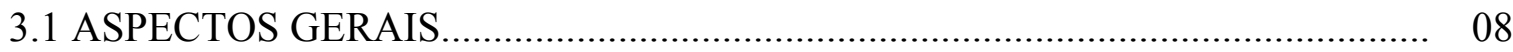

3.2 ETAPAS DO PROCESSO INDUSTRIAL DO ÁLCOOL ..................................... 10

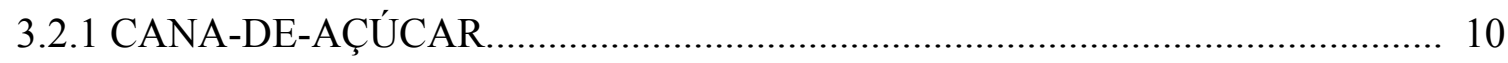

3.2.2 RECEPÇÃO DA CANA-DE-AÇÚCAR .................................................... 10

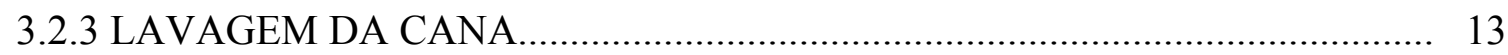

3.2.4 PREPARAÇÃO DA CANA..................................................................... 14

3.2.5 EXTRAÇÃO DO CALDO DA CANA....................................................... 15

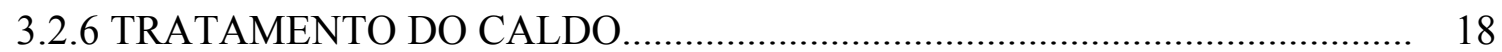

3.2.6.1 PENEIRAMENTO DO CALDO ............................................................ 18 


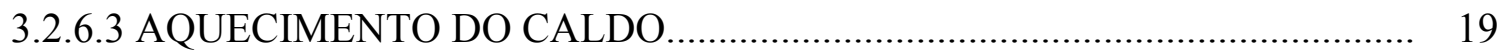

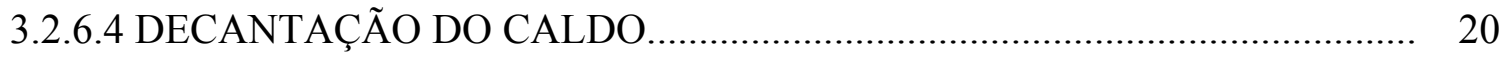

3.2.6.5 FILTRAÇÃO ................................................................................ 21

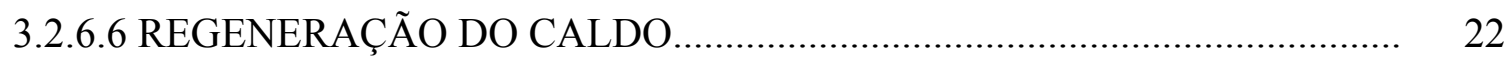

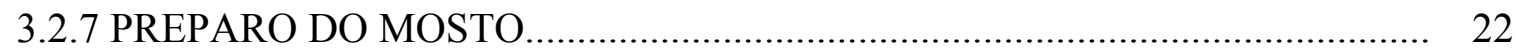

3.2.7.1 PREPARO DO FERMENTO ................................................................... 23

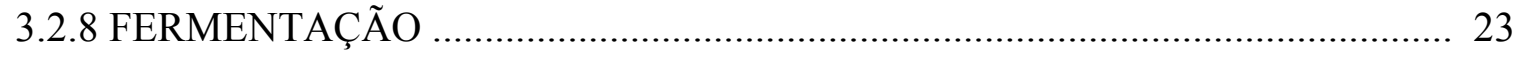

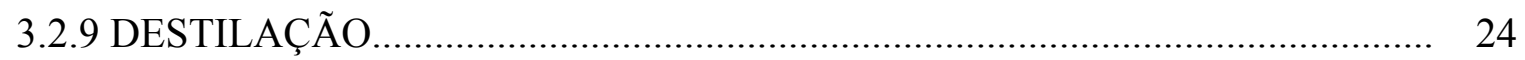

3.3 EXERGIA (ou DISPONIBILIDADE DE ENERGIA) e QUALIDADE DE

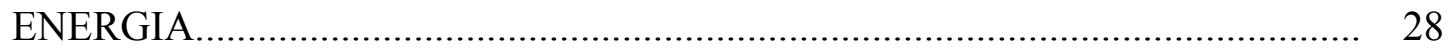

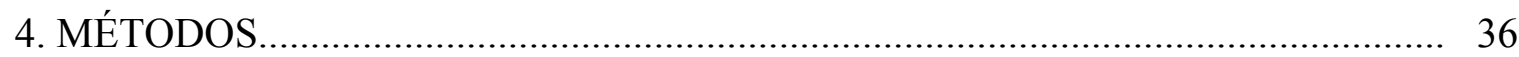

4.1 O PROCESSO INDUSTRIAL DO ÁLCOOL E OS EFLUENTES

GERADOS

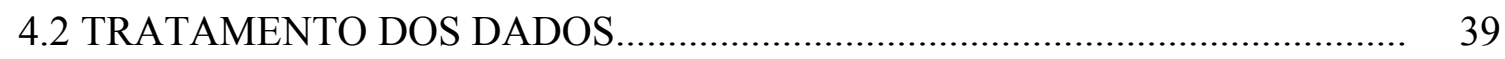

4.3 MÉTOdOS DE CÁLCULO...................................................................... 39

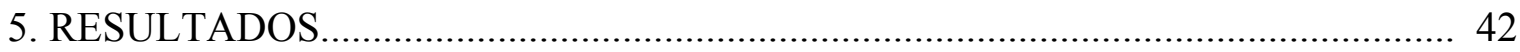

5.1 RESULTADOS DAS CARACTERIZAÇÕES.................................................... 42

5.1.1 CARACTERIZAÇÃO DA UNIDADE INDUSTRIAL I............................... 43

5.1.2 PROCESSO INDUSTRIAL DESENVOLVIDO NA UNIDADE I ................ 43

5.1.3 CARACTERIZAÇÃO DA UNIDADE INDUSTRIAL II .............................. 48

5.1.4 PROCESSO INDUSTRIAL NA UNIDADE INDUSTRIAL II ...................... 49

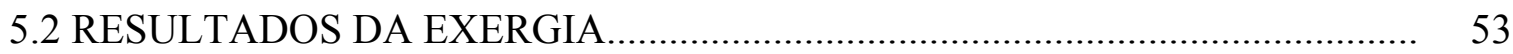

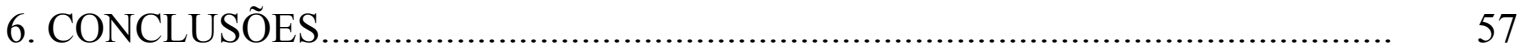

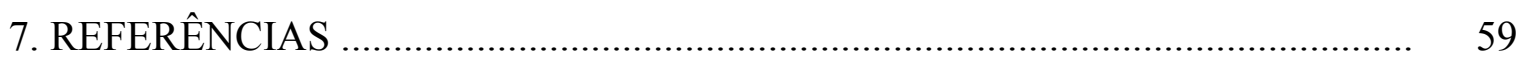

